\title{
Research of circuit breaker intelligent fault diagnosis method based on double clustering
}

\author{
He Mengyuan, Ding Qiaolin, Zhao Shutao, and Wei Yao \\ North China Electric Power University, Baoding 071003, China
}

\begin{abstract}
According to the energy variation of the mechanical transmission in the process of circuit breaker operation which is characterized by acoustic and vibration signals, a new method of high Voltage circuit breaker mechanical fault diagnosis was proposed in this paper. This method combined Density Peaks Clustering Algorithm (DPCA) fused Kernel Fuzzy C Means (KFCM) and support vector machine (SVM). It is an intelligent method of double clustering. Vibration and acoustic signals are decomposed by Local Mean Decomposition. Three product function components with the largest correlation of the original signal are filtered. And the characteristic entropy can be extracted by approximate entropy. DPCA is utilized to get the best peak density clustering decision and optimize the initial clustering center of KFCM. The fault training samples is pre-classified and input SVM. And the fault classification result of the circuit breaker can be received by mesh optimization algorithm. Finally, the DPCA-KFCM and SVM method in the fault diagnosis of the circuit breaker is verified by the typical failure test of the circuit breaker, the loosening of the pedestal and the refusal of the circuit breaker, which improve the accuracy of the fault diagnosis greatly.
\end{abstract}

Keywords: vibration and acoustic joint, kernel fuzzy c means, density peaks clustering algorithm, circuit breaker, fault diagnosis

Classification: Power devices and circuits

\section{References}

[1] J. Huang, et al:: "An intelligent fault diagnosis method of high voltage circuit breaker based on improved EMD energy entropy and multi-class support vector machine," Electr. Power Syst. Res. 81 (2011) 400 (DOI: 10.1016/j.epsr.2010. 10.029).

[2] B. Xie, et al.: "Partition of the development stage of air-gap discharge in oilpaper insulation based on wavelet packet energy entropy," IEEE Trans. Dielectr. Electr. Insul. 23 (2016) 866 (DOI: 10.1109/TDEI.2015.005430).

[3] N. Charbkaew, et al:: "Vibration signal analysis for condition monitoring of puffer-type high-voltage circuit breakers using wavelet transform," IEEJ Trans. Electr. Electron. Eng. 7 (2012) 13 (DOI: 10.1002/tee.21690).

[4] D. Pérez-Canales, et al:: "Identification of dynamic instabilities in machining process using the approximate entropy method," Int. J. Mach. Tools Manuf. 51 (2011) 556 (DOI: 10.1016/j.ijmachtools.2011.02.004). 
[5] A. Rodriguez and A. Laio: "Clustering by fast search and find of density peaks," Science 344 (2014) 1492 (DOI: 10.1126/science.1242072).

[6] D. Hu, et al.: "A novel KFCM based fault diagnosis method for unknown faults in satellite reaction wheels," ISA Trans. 51 (2012) 309 (DOI: 10.1016/ j.isatra.2011.10.005).

[7] M. Vatsa, et al.: "Improving biometric recognition accuracy and robustness using DWT and SVM watermarking," IEICE Electron. Express 2 (2005) 362 (DOI: 10.1587/elex.2.362).

[8] S.-K. Kim and J.-H. Chang: "Speech/music classification enhancement for 3GPP2 SMV codec based on support vector machine," IEICE Trans. E92.A (2009) 630 (DOI: 10.1587/transfun.E92.A.630).

[9] H. Feng, et al:: "WLAN traffic prediction using support vector machine," IEICE Trans. E92.B (2009) 2915 (DOI: 10.1587/transcom.E92.B.2915).

[10] Y. X. Su, et al.: "EEMD-ApEn applied in power quality detection and classification," Appl. Mater. Technol. Modern Manuf. 423-426 (2013) 2443 (DOI: 10.4028/www.scientific.net/AMM.423-426.2443).

\section{Introduction}

Circuit breaker is important for control and protection in power system. With the development of smart grid construction, the demand on the reliability of the circuit breaker is getting higher and higher. Main working reliability of the circuit breaker is determined by its mechanical properties. According to the statistics, malignant accidents caused by mechanical failure such as refusing action, error action and delay switch-off account for more than $80 \%$ of total accident, which resulted from the operating mechanism failure account for $64.8 \%$. Therefore, the current circuit breaker fault diagnosis research mainly revolves around operator mechanical failure.

Extract the vibration signal energy entropy is often adopted in mechanical fault diagnosis of circuit breaker as an input vector to identification methods such as expert system and artificial neural network. Both of wavelet analysis and Empirical Mode Decomposition (EMD) can realize the feature analysis of vibration signal $[1,2,3]$. Wavelet analysis method is simple and easy to implement, but can't decompose the signal adaptively, and the optimal selection of wavelet basis is complicated. EMD plays the role of the bridge for the meaningful Hilbert transform of non-stationary signal, but it will produce the negative frequency which cannot be explained. In addition, the expert system needs a large number of accumulation of experience, different rules has a great influence on the final result. For Neural network, accuracy of fault identification is directly related to the number of training sample. However, opening and closing operation of the circuit breaker rarely leads to the lack of samples which affected the accuracy of fault identification in different extent.

Vibration and acoustic joint diagnosis become an emerging research direction of high voltage circuit breaker mechanical failure. In view of the vibration and acoustic signals have the characteristics of nonlinear and non-stationary, reasonable selection of signal characteristics and fusion algorithm is the key to fault diagnosis. Local Mean Decomposition (LMD) compared to EMD is improved for instanta- 
neous frequency and envelope method, especially for differentiation degrees of processing effect in high frequency signal is higher than the EMD, and it can reflect the characteristics of the fault more clearly. Approximate entropy [4] is a measurement method of the statistical time sequence complexity based on marginal probability distribution. It doesn't require long length data, and has a strong antinoise ability which can be used as the characteristics of sound and vibration signal. Alex recently put forward a kind of spatial clustering algorithm based on density peak on SCIENCE [5] whose depiction of core idea is the clustering center. Initial clustering center of KFCM is optimized by the peak density clustering algorithm to use for control circuit fault of circuit breaker whose sample characteristics is approximate [6]. It can effectively reduce the clustering iterations, improve the clustering accuracy. Compared with neural network, the advantage of Support vector machine (SVM) $[7,8,9]$ is that only a small amount of learning samples can obtain better classification results. It is suitable for small sample situation in circuit breaker fault diagnosis operation.

\section{Characteristic extraction of the acoustic and vibration signal}

\subsection{Time-scale counterpoint}

Because of different artificial control speed and propagation speed in each collection when signal was acquired by vibration sensor and acoustic sensor, the time of signal collected before and after will have certain difference. As shown in Fig. 1.

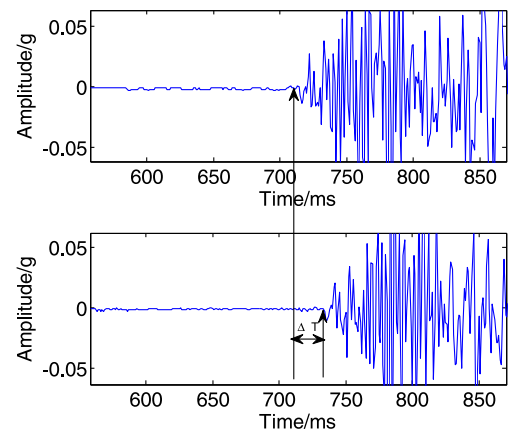

Fig. 1. Two vibration signal time lag $\Delta \mathrm{T}$ schematic diagram

Minkowski formula is used in envelope signal of acoustic and vibration. Such as type (1). According to $p$ root of the sum of $p$ power of the absolute difference between two variables to judge the similarity and find the similarity of the highest moment. Through dislocation for the acoustic signal, the acoustic signal benchmark starting point can be obtained by the revision. Therefore, the time-scale of the vibration and acoustic signal can be counterpointed accurately.

$$
M_{p}(x, y)=\left(\sum_{i, j=1}^{n}\left|x_{i}-y_{j}\right|^{p}\right)^{1 / p}
$$

Where $\mathrm{x}$ and $\mathrm{y}$ are $\mathrm{N}$-dimensional samples, $\mathrm{p}$ is distance adjustment parameters. It is represented as Euclidean distance when $\mathrm{p}$ is equal to 2. The characterization is the shortest distance between the data. 


\subsection{Local mean decomposition}

LMD method is developed on the basis of EMD decomposition. Similar to EMD, both of them are based on the extreme value point to define the local mean value function and the local envelope function. However, smoothing method is adopted by LMD to form local mean value function and local envelope function, it can avoid over envelope and owe envelope phenomenon caused by EMD during the upper and lower envelope is formed in cubic spline function. LMD can isolate pure frequency modulation signal and envelope signal from complex multi-component signal. According to multiplication, the sum of a series of Production Function (PF) whose instantaneous frequency has a physical meaning and a monotonic Function can be obtained:

$$
\mathrm{x}(\mathrm{t})=\sum_{\mathrm{p}=1}^{\mathrm{k}} \mathrm{PF}_{\mathrm{p}}(\mathrm{t})+\mathrm{u}_{\mathrm{k}}(\mathrm{t})
$$

Circulation to release all PF components can get time-frequency distribution of the original signal.

\subsection{Approximate entropy}

Approximate Entropy (ApEn) uses a negative number to represent the signal sequence complexity and irregularity. The more complexity of the signal, the bigger approximate entropy will have. It can use a short data to estimate Approximate Entropy of the signal. And it has certain anti-interference ability and a good tolerance capacity to the transient signal. It can generalize a relatively short data containing noise to a stable statistic. After signal decomposition of LMD, PF component is shown as a time series in turn from high frequency to low frequency. Therefore, approximate entropy is adopted to quantify the PF component.

It can realize the extraction of the useful information with the target of complexity of the PF component. The specific algorithm steps can be found in the literature [10].

\section{DPCA-KFCM-SVM circuit fault diagnosis method}

\subsection{Density peaks clustering algorithm}

Density Peaks Clustering Algorithm (DPCA) is a spatial Clustering Algorithm based on density. The main goal is to find high density area which is separated by low density area. The core idea is to depict the clustering center.

A sample is set as $\mathrm{X}=\left(x_{1}, x_{2}, \ldots, x_{n}\right)$, the local density $\rho_{\mathrm{i}}$ of the sample $\mathrm{x}_{\mathrm{i}}$ is defined as:

$$
\rho_{\mathrm{i}}=\sum_{\mathrm{j}=1} \varphi\left(\mathrm{d}_{\mathrm{ij}}-\mathrm{d}_{\mathrm{c}}\right)
$$

$\varphi(\mathrm{x})=\left\{\begin{array}{ll}1, & \mathrm{x}<0 \\ 0, & \mathrm{x} \geq 0\end{array}, \mathrm{~d}_{\mathrm{c}}\right.$ is truncated distance which should be specified in advance, $d_{i j}$ is the Euclidean distance of the sample $x_{i}$ and $x_{j}$.

The distance $\delta_{\mathrm{i}}$ of sample $\mathrm{x}_{\mathrm{i}}$ is defined as:

$$
\begin{cases}\delta_{\mathrm{i}}=\min _{\mathrm{j}: \rho_{\mathrm{j}}>\rho_{\mathrm{i}}}\left(\mathrm{d}_{\mathrm{ij}}\right), & \mathrm{i} \geq 1 \\ \delta_{\mathrm{i}}=\max _{\mathrm{j}}\left(\mathrm{d}_{\mathrm{ij}}\right), & \rho_{\mathrm{i}} \text { as the top global }\end{cases}
$$


Through calculation of every point in the sample set above, you can get $\left(\rho_{\mathrm{i}}, \delta_{\mathrm{i}}\right)$. Decision diagram can be obtained by drawing $\left(\rho_{\mathrm{i}}, \delta_{\mathrm{i}}\right)$ of each point in plane coordinate (with local density on the horizontal axis, the distance on the vertical axis). Those points with larger distances and larger local density at the same time are defined as the cluster center.

\subsection{Kernel fuzzy C means}

KFCM brings the idea of the kernel function into FCM. Kernel function takes the place of Euclidean distance in the traditional FCM algorithm. KFCM mainly uses for scale transform. It maps the samples from the $\mathrm{R}^{\mathrm{p}}$ space to high dimension space $\mathrm{R}^{\mathrm{q}}$ by kernel function. Thereby the difference degree between categories can be increased, and bad treatment of fuzzy category in the classic FCM is overcame. KFCM has less iteration steps and can draw the same pattern of clustering. The clustering of KFCM can improve the efficiency of clustering.

The objective function of KFCM is the kernel space form of FCM, namely

$$
\mathrm{J}_{\mathrm{m}}(\mathrm{U}, \mathrm{V})=\sum_{\mathrm{i}=1}^{\mathrm{c}} \sum_{\mathrm{k}=1}^{\mathrm{n}} \mathrm{u}_{\mathrm{ik}}^{\mathrm{m}}\left\|\emptyset\left(\mathrm{x}_{\mathrm{k}}\right)-\emptyset\left(\mathrm{v}_{\mathrm{i}}\right)\right\|^{2}
$$

Ø: nonlinear mapping, $X=\left\{\mathrm{x}_{1}, \mathrm{x}_{2}, \ldots, \mathrm{x}_{\mathrm{n}}\right\} \in \mathrm{R}^{\mathrm{p}}$ : sample set, $\mathrm{U}=\left[\mathrm{u}_{\mathrm{ik}}\right]_{\mathrm{c} \times \mathrm{n}}(\forall \mathrm{i}, \mathrm{k}$, $\left.\mathrm{u}_{\mathrm{ik}} \in[0,1] ; \forall \mathrm{k}, \sum_{\mathrm{i}=1}^{\mathrm{c}} \mathrm{u}_{\mathrm{ik}}=1 ; \forall \mathrm{i}, 0<\sum_{\mathrm{k}=1}^{\mathrm{n}} \mathrm{u}_{\mathrm{ik}}<n\right)$ : fuzzy classified matrix $\mathrm{V}=$ $\left\{\mathrm{v}_{1}, \mathrm{v}_{2}, \ldots, \mathrm{v}_{\mathrm{c}}\right\}$ : clustering center vector set of class $\mathrm{c}, \mathrm{m}>1$ : Fuzzy weighted exponent, $\quad\left\|\emptyset \mathrm{x}_{\mathrm{k}}-\emptyset\left(\mathrm{v}_{\mathrm{i}}\right)\right\|^{2}=\mathrm{K}\left(\mathrm{x}_{\mathrm{k}}, \mathrm{x}_{\mathrm{k}}\right)+\mathrm{K}\left(\mathrm{v}_{\mathrm{i}}, \mathrm{v}_{\mathrm{i}}\right)-2 \mathrm{~K}\left(\mathrm{x}_{\mathrm{k}}, \mathrm{v}_{\mathrm{i}}\right), \quad \mathrm{K}(\mathrm{x}, \mathrm{y})=$ $\emptyset(\mathrm{x})^{\mathrm{T}} \emptyset(\mathrm{y})$ : kernel function.

In the above constraints, the objective function can be optimized as:

$$
\begin{aligned}
u_{i k} & =\frac{\left\{1 /\left[K\left(x_{k}, x_{k}\right)-2 K\left(x_{k}, v_{i}\right)+K\left(v_{i}, v_{i}\right)\right]\right\}^{1 /(m-1)}}{\sum_{i=1}^{c}\left\{1 /\left[K\left(x_{k}, x_{k}\right)-2 K\left(x_{k}, v_{i}\right)+K\left(v_{i}, v_{i}\right)\right]\right\}^{1 /(m-1)}} \\
v_{i} & =\sum_{k=1}^{n} u_{i k}^{m} K\left(x_{k}, v_{i}\right) x_{k} / \sum_{k=1}^{n} u_{i k}^{m} K\left(x_{k}, v_{i}\right)
\end{aligned}
$$

\subsection{DPCA-KFCM-LIBSVM algorithm}

KFCM has the problem of random initial clustering center that cannot obtain the global optimal and prone to consistency when the center of clustering is close or overlap. DPCA is introduced in KFCM algorithm in this paper. DPCA and KFCM are organic unified in together, DPCA was used to optimize the initial clustering center and overcome the premature problem of single KFCM algorithm so as to seek a better clustering effect.

The algorithm process is:

By observing and constructing decision diagram of sample distance relative to the sample density, peak points in decision diagram can be chosen as the initial clustering center. The peak points have both of the high sample density and high distance in decision diagram, and they are obviously far from most of the sample points of the data sets. The maximum optimal individual fitness can be accepted. Iteratively updating and comparing for the location and fitness constantly, until meet the termination conditions. The global optimal individuals can be obtained 
and defined as the best clustering center. Maximum membership principle is used degree judge the sample fault category, and the optimization process is ended up. On this basis, establish multiple SVM trainers and input the sampling characteristics data to the trainers, the comprehensive evaluation of the final diagnosis results are obtained.

\section{Implementation scheme of vibration and acoustic joint circuit breaker fault diagnosis}

In this paper, LMD approximate entropy is used to extract the state characteristics of acoustic and vibration signals. In SVM training phase, the maximum density peak decision of DPCA is used to optimization the initialize clustering center of KFCM. Preliminary classification is used for training data through fuzzy kernel clustering to obtain the least risky optimal hyperplane. In circuit breaker fault identification stage, characteristics of the acoustic and vibration signal test samples are input to SVM. And the grid optimization algorithm is used to obtain the highest classification accuracy for further split and get classification accuracy of each fault. The overall scheme of the circuit breaker fault diagnosis is shown in Fig. 2.

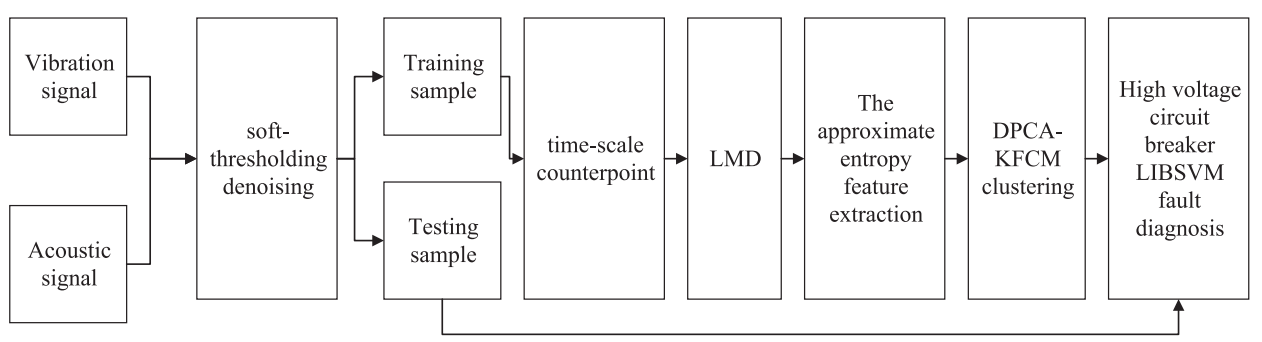

Fig. 2. Overall scheme of the circuit breaker fault diagnosis

Vibration and acoustic signals are synchronous acquired in the process of high voltage circuit breaker operation. Signals are denoised and divided into training sample and test sample sets. Time-scale counterpoint is used for each acoustic signal and vibration signal acquired before and after by Minkowski formula to eliminate the time lag. LMD is used in sections to get PF matrix composed of PF component. Approximate entropy of each PF component of the PF matrix is calculated as the state characteristic of the high voltage circuit breaker. Preliminary classification is carried out on the training sample by KFCM based on DPCA optimization to establish the membership degree of mapping between data samples and fault types. And on this basis, Multiple SVM trainer is established. After the test samples are input to trainer, the comprehensive evaluation of the final diagnosis results can be obtained.

\section{Test and result analysis}

\subsection{The test process}

$\mathrm{ZN}-65$ vacuum circuit breaker is used as the experiment platform to analog three 
simulated by a corner of the circuit breaker is pad high as shown in Fig. 3(b). And the refusal of the circuit breaker is simulated by adjust the core gap makes the first quantum cannot be firing from dynamic as shown in Fig. 3(c). The numbers of the fault categories are recorded as $F_{1}$ for normal, $F_{2}$ for spindle jamming, $F_{3}$ for pedestal looseness, $\mathrm{F}_{4}$ for the refusal of the circuit breaker.

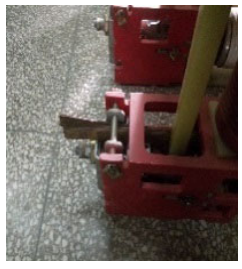

(a) Spindle jamming

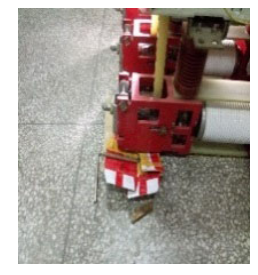

(b) Pedestal looseness

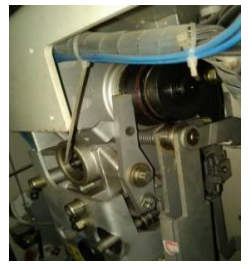

(c) Refusal

Fig. 3. Three faults schematic

PXI system is adopted in experiments to complete the acquisition of the circuit breaker fault signal. Sensor is connected to PXI synchronous acquisition card by $50 \Omega$ coaxial cable. IEPE method and anti-aliasing filter are adopted in acquisition card whose signal has $114 \mathrm{db}$ dynamic range. Through the proper grounding and shielding, interference can be reduced to a minimum.

In the experiment, the high fidelity cartridge bullet pin-socket type noise detector is adopted to acquire the acoustic signal acquisition. It is equipped with AGC automatic gain control circuit to enhance the distance sound wave. Its impact range can reach up to 100 square meters, and sensitivity can reach $-46 \mathrm{DdB}$. The frequency ranges from 20 to $20 \mathrm{KHZ}$. The piezoelectric acceleration sensor is adopted to acquire the vibration signal. It has the characteristics of wide frequency response, high sensitivity, small transverse sensitivity and strong anti-interference ability. The vibration sensor was fixed on the bracket of the circuit breaker by bolts, and high-fidelity pick-up was placed on the equidistant around $20 \mathrm{~cm}$ position of the circuit breaker. Break-brake coil current signal was used as a trigger source to collection card. Thirty times break-brake experiments are performed in total, including normal break-brake for 13 times, spindle jamming fault for 6 times, 6 times in the pedestal looseness fault, 5 times in break-brake refusing action.

\subsection{Signal feature extraction}

After the soft threshold denoising processing of the collected acoustic signal, signal waveform of the normal and fault condition are shown in Fig. 4.

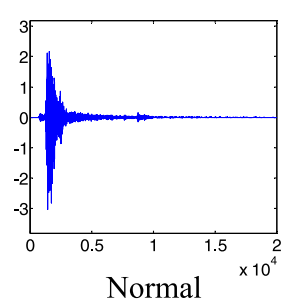

(a) Vibration Signal
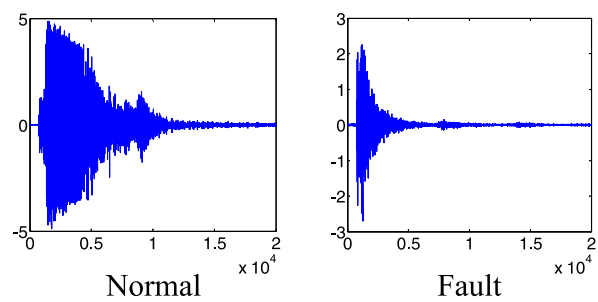

(b) Acoustic Signal 
LMD was used for signal decomposition, and time-scale counterpoint for the acoustic and vibration signal. Take example for normal signal, the decomposition result is shown in Fig. 5. Eight PF components and a remaining component were decomposed. From the decomposition result, the first three PF components contain most of the original signal information. At the same time, since multi-component will increase the computational complexity, which will reduce the operation speed and the efficiency of diagnosis. Thus the first three PF components were selected to obtain the approximate entropy. And the approximate entropy was acquired as the feature vector. Part approximate entropy data of the fault type is shown in Table I.

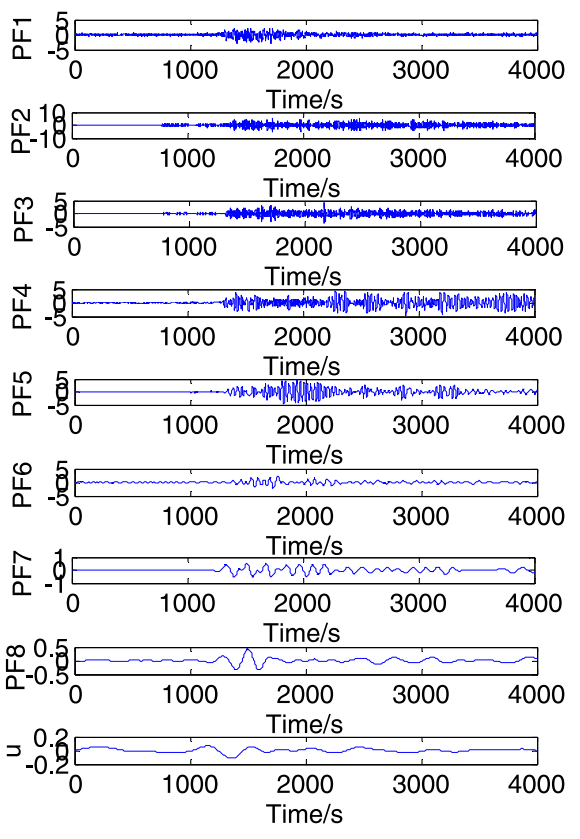

(a) PF component of acoustic signal

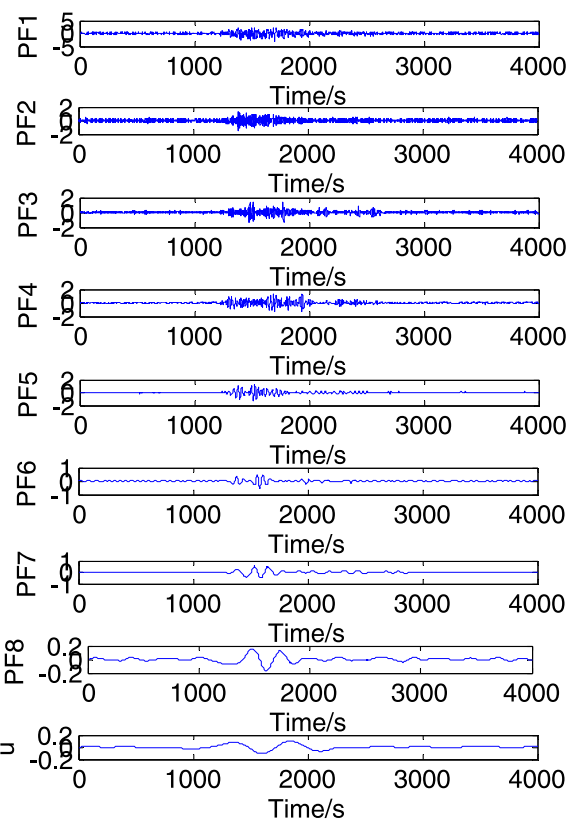

(b) PF component of vibration signal

Fig. 5. PF component of opening vibration and acoustic signals in normal state

Table I. The approximate entropies of the three PF components of four kinds of vibration and acoustic

\begin{tabular}{ccccc}
\hline $\begin{array}{c}\text { Signal } \\
\text { type }\end{array}$ & Fault type & PF1 & PF2 & PF3 \\
\hline \multirow{2}{*}{$\begin{array}{cccc}\text { Vibration } \\
\text { signal }\end{array}$} & Normal & 0.0812 & 0.1178 & 0.0739 \\
& Reoseness & 0.0923 & 0.1080 & 0.0779 \\
& Jamming & 0.0835 & 0.1205 & 0.0728 \\
Acoustic & Normal & 0.0797 & 0.1205 & 0.0728 \\
signal & Looseness & 0.1009 & 0.0908 & 0.0847 \\
& Refusal & 0.0757 & 0.1145 & 0.0763 \\
& Jamming & 0.1026 & 0.0945 & 0.0822 \\
\hline
\end{tabular}

\subsection{The fault diagnosis result analysis}

The training sample set is randomly selected from $70 \%$ of the four high voltage circuit breaker fault state sample as normal, spindle jamming, pedestal looseness 
fault and refusing. While the corresponding remaining samples are taken as the test sample set for testing diagnostic accuracy.

The peak density clustering decision diagram is shown in Fig. 6. In Fig. 6, four points of the upper right corner are selected for the initial clustering center. The training sample set is analyzed by KFCM clustering algorithm based on DPCA, and the clustering results are shown in Fig. 7.

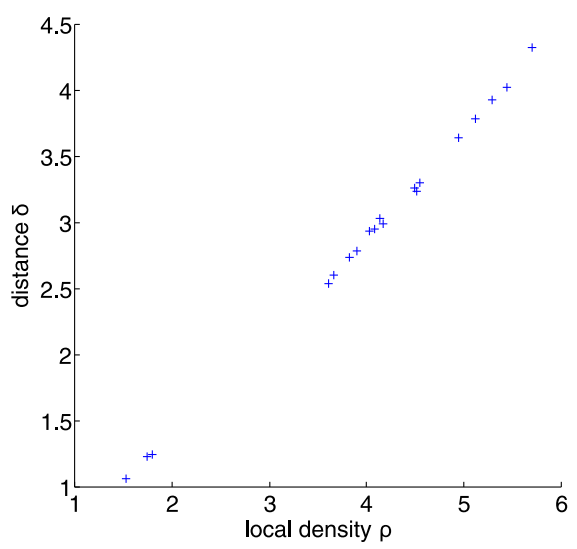

Fig. 6. Peak density clustering decision diagram

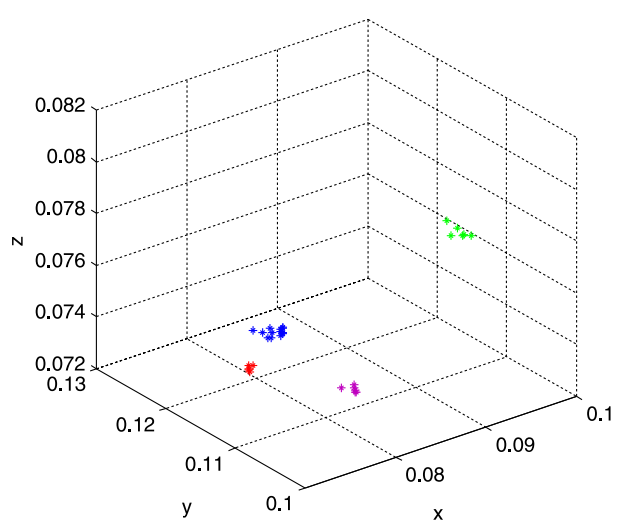

Fig. 7. Clustering results in three dimensional space

The clustering results are used to train SVM and obtain the SVM classifier. After inputting the test sample sets into SVM, through the grid search to find the optimal parameters of SVM and the highest classification accuracy in CV sense. SVM fault diagnosis results are obtained. In addition, it is compared with FCMSVM and KFCM-SVM methods for fault classification test. Test results are shown in Table II. It can be seen from Table II that FOA-KFCM-SVM has higher accuracy in the process of brake vibration signal data for fault diagnosis. 
Table II. Comparison of different algorithms diagnose performance

\begin{tabular}{cc}
\hline Methods & Diagnostic accuracy \\
\hline FCM-SVM & $80 \% \%$ \\
KFCM-SVM & $92.3077 \%$ \\
DPCA-KFCM-SVM & $99.9841 \%$ \\
\hline
\end{tabular}

\section{Conclusions}

This paper puts forward a kind of circuit breaker intelligent fault diagnosis methods with vibration and acoustic joint based on double clustering. Four kinds of typical states are acquired for analysis. The four states contain normal state, spindle jamming, pedestal looseness and refusing action. The conclusions are as following:

1) Time-scale counterpoint can eliminate the time lag of the signal acquired before and after, and fix the sensitivity of DPCA to the sample time. LMD and approximate entropy are used to extract the feature vector, it can solve the problem of over and owe envelope phenomenon of EMD method.

2) DPCA is used to optimize the initial clustering center of KFCM, which can effectively reduce the clustering iterations, shortened the time clustering. Combine DPCA-KFCM with SVM can greatly solve the problems such as small sample, nonlinear and high dimension of the circuit breaker status identification. It can improve the accuracy of circuit breaker failure diagnosis.

3) This paper only to incorporate acoustic and vibration signal. It can also add other signals in actual application with the method in this paper. For example, closing coil current signal, voltage signal, the oil pressure signal of hydraulic mechanism and so on. Fusion of multiple signal analysis in circuit breaker fault diagnosis is one of the important developing directions of the future research.

4) The operation environment of high voltage circuit breaker is complex. And the fault types are various. This paper simulates only several typical mechanical faults. In the follow-up work, different types of the typical fault data samples need to be collected as much as possible. At the same time, the fault simulation test should be made further research. For the application of actual industrial, it is necessary to continuously improve the function of software and hardware, and strengthen the practical engineering application of existing theoretical research results. 